\title{
8BOCEHMO
}

ISSN: $2447-8504$

DOI: $10.30938 /$ bocehm.v8i23.4972

\section{CONSIDERAÇÕES INICIAIS SOBRE A OBRA KITAB AL-FUSUL FI AL-HISAB AL-HINDI}

\section{INITIAL CONSIDERATIONS ON THE ORIGINAL WORK KITAB AL-FUSUL FI AL- HISAB AL-HINDI}

\author{
Suziê Maria de Albuquerque ${ }^{1}$; Bernadete Barbosa Morey²
}

\begin{abstract}
RESUMO
O presente artigo faz parte da etapa inicial de uma pesquisa de doutorado em história da matemática islâmica que estuda o texto original Kitab al-Fusul fi al-Hisab al-Hindi na versão de 1186 (E.C.), escrita por Abu al-Hasan Almad ibn Ibrahim al-Uqlidisi na cidade de Damasco na Síria.Podendo assim revelar indícios do contexto histórico da sociedade na qual foi produzido. Diante disso, elencou-se como objetivo conhecer de modo preliminar os elementos estruturais desse texto. Para tanto, realizou-se um estudo documental da versão traduzida do árabepara o inglês no ano de 1978. A leitura prévia da obra permitiu identificar o movimento de conhecimentos matemáticos por meio do encontro da aritmética indiana com a árabe. Esse indício se revelou pela estrutura da obra na qual compila os conhecimentos indianos de duplicação, redução, multiplicação, divisão, multiplicação e divisão com frações, cálculo de raízes, dentre outros realizados no ábaco de areia. De posse desses conhecimentos, Al-Uqlidisi inclui sua contribuição, sistematizando os procedimentos para a realização dessas contas em uma espécie de papel da época. A compreensão do conteúdo e objetivo da organização de cada livro pode favorecer o aprofundamento das análises contextuais, historiográfica e epistemológicas a serem realizadas.
\end{abstract}

Palavras-chave:Aritmética de al-Uqlidisi; Hisab al-Hindi; História da Matemática.

\begin{abstract}
This article is part of the initial stage of a doctoral research in the history of Islamic mathematics that studies the original text Kitab al-Fusul fi al-Hisab al-Hindi in the version of 1186 (EC), written by Abu al-Hasan Almad ibn Ibrahim al-Uqlidisi in the city of Damascus in Syria, thus being able to reveal clues to the historical context of the society in which it was produced. In view of this, it was listed as an objective to know in a preliminary way the structural elements of this text. To this end, a documentary study of the translated version from Arabic into English was carried out in 1978. The previous reading of the work made it possible to identify the movement of mathematical knowledge through the encounter of Indian arithmetic with Arabic. This evidence was revealed by the structure of the work in which it compiles the Indian knowledge of duplication, reduction, multiplication, division, multiplication and division with fractions, calculation of roots, among others carried out in the sand abacus. With this knowledge, Al-
\end{abstract}

\footnotetext{
${ }^{1}$ Mestra pelo Instituto Federal de Educação, Ciência e Tecnologia do Estado do Ceará (IFCE). Doutoranda em Ensino de Ciências e Matemática pela Universidade Federal do Rio Grande do Norte (UFRN), Natal, Rio Grande do Norte, Brasil. E-mail: suziealbuquerque@ hotmail.com.

(iD ORCID iD:https://orcid.org/0000-0002-2531-0385.

2 Doutora pela Universidade Amizade dos Povos (SIGLAUAP-Moscou). Prof. Colaboradora Aposentada e na Universidade Federal do Rio Grande do Norte (UFRN), Natal, Rio Grande do Norte, Brasil. E-mail: bernadetemorey@gmail.com.
}

(DORCID iD: https://orcid.org/0000-0003-3253-0383 
Uqlidisi includes his contribution, systematizing the procedures for the realization of these accounts in a kind of paper of the time. The understanding of the content and objective of the organization of each book can favor the deepening of the contextual, historiographical and epistemological analyzes to be carried out.

Keywords: Al-Uqlidisiarithmetic; Hisab al-Hindi; HistoryofMathematics. 


\section{Introdução}

As discussões em torno do sistema de numeração utilizado no mundo islâmico conduz a uma viagem no tempo, na busca dos símbolos, métodos e instrumentos de cálculo que proporcionaram o desenvolvimento de uma aritmética voltada para a solução de problemas práticos como a cobrança de imposto, divisão de heranças, realização de cálculos astronômicos, dentre outros. Essas demandas emergiram da expansão territorial e comercial dos povos islâmicos na chamada idade de ouro, entre os séculos VIII e XIII.

Nesse período, sob o domínio dos califas, foi incentivado o desenvolvimento das ciências e do comércio nas regiões governadas, bem como a aquisição e produção de cópias para o idioma árabe de fontes de conhecimento advindas dos gregos e indianos, como destaca Abel (1935). Entretanto, além de preservar esses conhecimentos estrangeiros, os árabes acrescentavam informações adicionais que complentariam o material adquirido, incorporando assim novas compreensões (TUNER, 2006).

Uma das obras em árabe que revelam esse contexto é o Kitab al-Fusul fi al-Hisab al-Hindi, escrito originalmente no ano 341 da Hégira ${ }^{3}$ (952 E.C.) na cidade de Damasco, por Abu al-Hasan Almad ibn Ibrahim al-Uqlidisi. Duas versões da referida obra foram localizadas nesta pesquisa, a primeira, manuscrita em árabe datada do ano de 1186 (E.C.).

Outra edição desse texto, traduzida a partir da produção do século XII para o inglês por Saidan (1978). O tradutor, historiador das matemáticas árabes medievais, tendo a análise da obra de Al-Uqlidisi como resultado de sua pesquisa doutoral na Universidade de Cartum no Sudão. Desenvolveu diversos estudos na área da história da matermática que lhe renderam publicações em revistas insternacionais como na Ísis da Universidade de Oxford. Além disso, lecionou em diversas faculdades e universidades árabes na Palestina, Sudão e Jordânia ${ }^{4}$. Conferindo assim credibilidade na produção da tradução, pois o responsável se trata de um estudioso especializado em estudos da história da matemática árabe.

Sobre o autor medieval não foram localizados registros bibliográficos, apenas uma possível interpretação para o significado do seu nome, proferida por Saidan (1966) na qual remete "al-Uqlidisi" a "o Euclidiano", aquele que dominava os ensinamentos da geometria de Euclides ou até mesmo se dedicava no trabalho de copista desses textos.

\footnotetext{
${ }^{3}$ Fuga de Maomé de Meca para Medina no ano de 622 (E.C.), marcando o início do calendário islâmico.

${ }^{4}$ VideAbattouy (2009)
} 
$\mathrm{Na}$ aritmética em específico, a obra mencionada trata dos conhecimentos indianos na qual são apresentados os símbolos numéricos indianos e os procedimentos operatórios deste povo que eram adotados na cidade de Damasco no século X. Além disso, explicita as contribuições islâmicas que redimensionaram as técnicas e instrumentos de cálculo indiano, imprimindo praticidade na realização das contas. $\mathrm{O}$ encontro de culturas no referido texto indica o movimento de elaboração, transmissão e transformação de conhecimentos matemáticos nesse espaço e tempo histórico, tendo em vista que a obra de Al-Uqlidisi é a mais antiga preservada no idioma original.

Diante disso, elencou-se como objetivo conhecer de modo preliminar os elementos estruturais desse texto. Para tanto, foi realizado um estudo documental a partir da seleção da versão em inglês, tendo em vista a facilidade com o idioma e dificuldade de ter acesso ao manuscrito original completo. Assim, foi realizada a leitura panorâmica da obra de Al-Uqlidisi, identificando os conhecimentos matemáticos presentes em seu corpo, abrindo precedentes para o levantamento de hipóteses sobre como estes se inserem no contexto histórico do passado.

\section{Kitab al-Fusul fi al-Hisab al-Hindi, de Abu al-Hasan Almadibn Ibrahim al-Uqlidisi}

O manuscrito árabeKitab al-Fusul fi al-Hisab al-Hindi na versão de 1186 (E.C.) foi conservado em microfilme na Biblioteca da mesquita YemiCami em Instambul.Parte deste arquivo consta digitalizado, liberado para download no Instituto de Manuscritos Árabes ${ }^{5}$ (Figura 1). A versão original de 952 (E.C.) bem como outras versões manuscritas além dessas não foram localizadas até o presente momento.

A partir do acesso à versão completa de Istambul, Saidan (1978) fez a tradução desse material para o inglês(Figura 3), realizando ainda um estudo com levantamento das discussões sobre essa aritmética proposta. No contato com texto medieval original, perceberam-se partes incompletas, como se o copista não tivesse repassado essas informações ou mesmo al-Uqlidisi não teria conseguido concluir toda a escrita desses ensinamentos. Essas são algumas hipóteses sobre as lacunas.

O tradutor diz ainda que omitiu algumas informações do frontispício da obra na tradução, sendo que estas correspondem às declarações de propriedade do manuscrito. 
Para o referido estudioso essas anotações poderiam não ser relevantes. Porém, nesta pesquisa realizada atualmente, essas passagens podem indicar elementos importantes do contexto histórico com relação à intencionalidade da produção do referido texto. Diante disso, posteriormente será analisado de forma minuciosa o frontispício da versão de alUqlidisi do ano de 1180.

Figura 1 -Frontispício da primeira versão em árabe da obra de Al-Uqlidisi

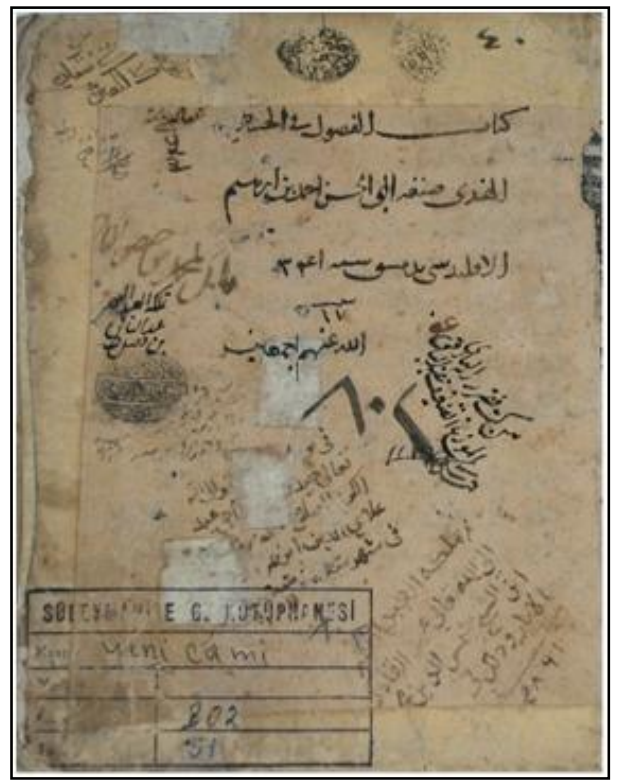

Fonte: Al-Uqlidisi (1186)

O trabalho de Saidan (1978) intitulado "The Arithmeticof Al-Uqlidisi: The Storyof Hindu-ArabicArithmetic as told in Kitab al-Fusul fi al-Hisab al-Hindi” é composto por três partes. Na primeira é apresentada uma introdução sobre as aritméticas islâmicas a partir do estudo de textos históricos. A segunda parte Saidan (1978) traz a tradução da obra de Al-Uqlidisi indicando no texto marcações de notas explicatórias que serão detalhadas na terceira parte como forma de comentários, contextualizado elementos do original árabe.

O Kitab al-Fusul fi al-Hisab al-Hindiem português "Livro de capítulos da aritmética indiana" pode fornecer indícios próximos da aritmética do período de sua escrita (século X), tendo em vista a preservação do idioma original. Além disso, a obra está organizada de maneira a explicitar os métodos de cálculo utilizados pelos indianos com a utilização do ábaco de poeira (takht) e a transposição desses conhecimentos para a realização dos cálculos operatórios no papel. Representando assim importante 
contribuição islâmica, contrariando a ideia de que estes teriam apenas perpetuado os conhecimentos de outros povos.

Figura 3 -Frontispício da tradução da obra de Al-Uqlidisi para o inglês

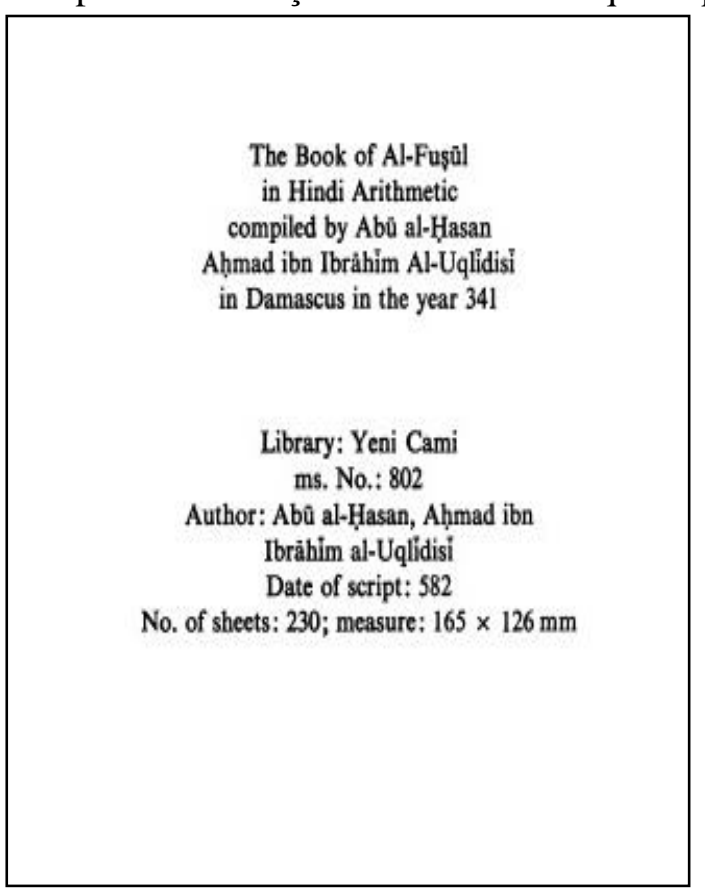

Fonte: Al-Uqlidisi (1978)

Saito (2015) enfatiza que a disseminação do sistema numérico indiano foi uma das maiores contribuições dos árabes ao Ocidente Latino, sobretudo a partir do século XII, período em que consta a cópia de al-Uqlidisi (1180). Entretanto, não foram encontrados indícios de que este material tenha alcançado o ocidente.

Apesar de propor o novo modo de registro dos números (no papel), o texto a que nos referimos indica uma coexistência de registros, o ábaco não foi totalmente excluído, tendo em vista que o cálculo no papel era algo novo e seria necessário adaptar os procedimentos adotados.O fluxo de circulação de conhecimento relatado pode ser observado a partir do conteúdo da obra descrito a seguir.

\section{O conteúdo da Obra de Aritmética de al-Uqlidisi}

A obra Kitab al-Fusul fi al-Hisab al-Hindi, de acordo com informações fornecidas por Al-Uqlidisi (1978), na sua apresentação, foi constituída a partir de consultas a outras obras de aritmética disponíveis no período que traziam os conhecimentos antigos 
(indianos, gregos e romanos), por indícios no texto. Porém, na apresentação deste manuscrito não constam os nomes dos autores consultados, mas é ressaltado que as mesmas foram comprovadas no contato com outros estudiosos do período em suas viagens.

O autor islâmico enfatiza ainda que a sua produção era a mais completa se comparada às anteriores, pois além de fazer a compilação das informações passadas, dos costumes de cálculo, ele as organiza de maneira que facilite a compreensão do aprendiz e ainda acrescenta os procedimentos para a realização dos cálculos substitituido o dispendioso uso do ábaco pelo papel.

A produção de uma obra que congregasse todos esses ensinamentos, com a preocupação de responder aos questionamentos do período sobre o sistema de numeração em uso e suas operações conduziu Al-Uqlidisi (1978) a organizar um total de quatro livros (Quadro 1). Cada uma dessas partes é formada por capítulos intitulados por conteúdos das operações na ordem adequada de serem ensinadas (AL-UQLIDISI, 1978).De fato, o autor tinha atenção para com os interessados em aprender esse ofício, indicando que este poderia desempenhar a função de ensinar, já que entendia da sequência adequada de introdução dos conteúdos para facilitar sua compreensão.

Quadro 1 - Conteúdo da obra Kitab al-Fusul fi al-Hisab al-Hindi

\begin{tabular}{|c|l|c|}
\hline ELEMENTO & \multicolumn{1}{|c|}{ CONTÉUDO } & PÁGINA \\
\hline Saudação & \multicolumn{1}{|c|}{ Em nome de Deus, o compassivo, o magnífico } & 35 \\
\hline Apresentação & Apresentação da obra e as motivações para a escrita & 35 \\
\hline \multicolumn{1}{|c|}{ LIVRO 1 } \\
\hline CAPÍTULO & \multicolumn{1}{|c|}{ TÍTULO } & PÁGINA \\
\hline 1 & Sobre as nove letras e os lugares & 41 \\
\hline 2 & Duplicando e reduzindo pela metade & 44 \\
\hline 3 & Em aumentar e diminuir & 46 \\
\hline 4 & Na multiplicação & 49 \\
\hline 5 & Na divisão & 65 \\
\hline 6 & Multiplicação de frações por números & 64 \\
\hline 7 & Combinando frações e partes & 67 \\
\hline 8 & $\begin{array}{l}\text { Sobre a multiplicação de um número com frações por } \\
\text { um número }\end{array}$ & 69 \\
\hline 9 & Multiplicando frações por frações & 72 \\
\hline 10 & $\begin{array}{l}\text { Sobre a multiplicação de um número com frações por } \\
\text { frações }\end{array}$ & 74 \\
\hline 11 & $\begin{array}{l}\text { Multiplicando um número com frações por um } \\
\text { número comfrações }\end{array}$ \\
\hline
\end{tabular}




\begin{tabular}{|c|c|c|}
\hline 12 & Em extraindo raízes de números, aberto e Asamm & 76 \\
\hline 13 & $\begin{array}{l}\text { Extraindo as raízes das frações, isoladas ou unidas a } \\
\text { Unidades }\end{array}$ & 80 \\
\hline 14 & Divisão em geral & 82 \\
\hline 15 & A Aritmética dos graus e os conceitos que envolve & 84 \\
\hline 16 & Reduzindo o grau pela metade e dobrando-o & 85 \\
\hline 17 & Sobre aumentar e diminuir & 87 \\
\hline 18 & Multiplicação de graus e minutos & 90 \\
\hline 19 & Sobre a divisão de graus e minutos & 93 \\
\hline 20 & Extraindo as raízes de graus e minutos & 96 \\
\hline 21 & Extraindo as raízes dos números Asamm & 99 \\
\hline \multicolumn{3}{|c|}{ LIVRO II } \\
\hline CAPÍTULO & TÍTULO & PÁGINA \\
\hline 1 & Duplicação & 104 \\
\hline 2 & Reduzindo pela metade & 108 \\
\hline 3 & $\begin{array}{l}\text { Redução pela metade onde as frações ocorrem e } \\
\text { duplicação - todos os métodos }\end{array}$ & 110 \\
\hline 4 & Aumentando, diferente do precedente (tipo) & 113 \\
\hline 5 & Na diminuição do tipo precedente & 115 \\
\hline 6 & $\begin{array}{l}\text { Sobre o tipo precedente de aumento e diminuição; } \\
\text { minuto (problemas) }\end{array}$ & 116 \\
\hline 7 & Esgotando um número por outro & 122 \\
\hline 8 & Consultas sobre as curiosidades de rejeição & 123 \\
\hline 9 & $\begin{array}{l}\text { Multiplicação de inteiros - todos os métodos: a } \\
\text { vertical e a ramificação }\end{array}$ & 127 \\
\hline 10 & Na multiplicação por (o método das) casas & 133 \\
\hline 11 & Curiosidades em multiplicação & 149 \\
\hline 12 & Na Divisão & 151 \\
\hline 13 & Na balança & 153 \\
\hline 14 & $\begin{array}{l}\text { Na multiplicação de inteiros e frações por inteiros e } \\
\text { frações, vertical e ramificação }\end{array}$ & 156 \\
\hline 15 & $\begin{array}{l}\text { Em que está mais, tais partes deste número ou tais } \\
\text { partes daquele? }\end{array}$ & 159 \\
\hline 16 & $\begin{array}{l}\text { Sobre a multiplicação de frações de frações de } \\
\text { frações }\end{array}$ & 161 \\
\hline 17 & Fazendo quadrados perfeitos para extrair suas raízes & 162 \\
\hline 18 & Extraindo a raiz do Asamm & 164 \\
\hline 19 & Extraindo as raízes do Asamm e testando-as & 166 \\
\hline 20 & $\begin{array}{l}\text { Sobre graus e minutos e os conceitos que eles } \\
\text { envolvem }\end{array}$ & 168 \\
\hline \multicolumn{3}{|c|}{ LIVRO III } \\
\hline CAPÍTULO & TÍTULO & PÁGINA \\
\hline 1 & $\begin{array}{l}\text { Justificações do hindi (aritmética) e seus porquês e } \\
\text { comos }\end{array}$ & 186 \\
\hline 2 & Consultas sobre duplicação e redução pela metade & 188 \\
\hline 3 & Consultas sobre aumento e diminuição & 189 \\
\hline
\end{tabular}


Suziê Maria de Albuquerque e Bernadete Barbosa Morey

Considerações iniciais sobre a obra Kitab AL-Fusul Fi Al-hisab Al-Hindi

\begin{tabular}{|c|c|c|}
\hline 4 & Consultas sobre multiplicação & 190 \\
\hline 5 & Consultas na divisão & 191 \\
\hline 6 & Consultas sobre raízes & 192 \\
\hline 7 & Verificação por balanceamento e suas justificativas & 195 \\
\hline 8 & Balanceamentos e frações & 197 \\
\hline 9 & Consultas sobre duplicação e redução pela metade & 202 \\
\hline 10 & Consultas sobre aumento e diminuição & 204 \\
\hline 11 & Consultas sobre adição de diferentes tipos & 206 \\
\hline 12 & Consultas sobre aumento e diminuição (vindo) juntos & 208 \\
\hline 13 & Curiosidades na multiplicação de nove & 211 \\
\hline 14 & Extraindo raízes por zeros onde as frações ocorrem & 213 \\
\hline 15 & Sobre a conversão de frações e partes, uma para outra & 215 \\
\hline 16 & Sobre a redução de frações e partes, uma da outra & 217 \\
\hline 17 & Consultas sobre minutos e suas partes de minutos & 219 \\
\hline 18 & Divisão pela vertical (método) & 224 \\
\hline 19 & Consultas na raiz (extração) pelo vertical (método) & 234 \\
\hline 20 & Consultas sobre lucro, envolvendo uma curiosidade & 237 \\
\hline 21 & $\begin{array}{l}\text { Sobre a justificativa de (o método de) extração do } \\
\text { lado do cubo }\end{array}$ & 242 \\
\hline \multicolumn{3}{|c|}{$\begin{array}{l}\text { LIVRO IV } \\
\end{array}$} \\
\hline CAPÍTULO & TÍTULO & PÁGINA \\
\hline 1 & O Método de Adição & 249 \\
\hline 2 & Na duplicação & 254 \\
\hline 3 & Na metade & 255 \\
\hline 4 & No acréscimo & 261 \\
\hline 5 & Na diminuição & 262 \\
\hline 6 & Na Multiplicação & 265 \\
\hline 7 & Na Divisão & 270 \\
\hline 8 & Sobre a multiplicação de frações por números & 276 \\
\hline 9 & Sobre Adicionar Frações e Partes & 277 \\
\hline 10 & $\begin{array}{l}\text { Sobre a multiplicação de um número com frações por } \\
\text { um número }\end{array}$ & 278 \\
\hline 11 & Sobre a multiplicação de frações por frações & 279 \\
\hline 12 & $\begin{array}{l}\text { Sobre a multiplicação de um número com frações por } \\
\text { frações }\end{array}$ & 280 \\
\hline 13 & $\begin{array}{l}\text { Multiplicando números inteiros e frações por } \\
\text { números inteiros e frações }\end{array}$ & 281 \\
\hline 14 & Geral, na Divisão & 282 \\
\hline 15 & Sobre a diferenciação entre frações & 285 \\
\hline 16 & $\begin{array}{l}\text { Sobre a conversão de frações e partes de um número } \\
\text { para outro número }\end{array}$ & 286 \\
\hline 17 & Sobre Extração de Raízes de Números & 287 \\
\hline 18 & Sobre extração de raízes onde ocorrem frações & 292 \\
\hline 19 & Nos Graus e Seus Esquemas & 294 \\
\hline 20 & Sobre Adicionar Graus, Minutos e Ordens Inferiores & 296 \\
\hline
\end{tabular}




\begin{tabular}{|c|l|c|}
\hline 21 & $\begin{array}{l}\text { Graus decrescentes, minutos e ordens inferiores uns } \\
\text { dos outros }\end{array}$ & 298 \\
\hline 22 & $\begin{array}{l}\text { Reduzindo Graus e Quaisquer Minutos e Segundos } \\
\text { que Cheguem a Seus Lugares Mais Baixos } \\
\text { Multiplicação de graus, minutos, segundos e ordens } \\
\text { inferiores, por ordens iguais ou diferentes }\end{array}$ & 299 \\
\hline 23 & $\begin{array}{l}\text { Sobre a Divisão de Graus e minutos e Ordens } \\
\text { Inferiores por Graus e minutos e Ordens Inferiores }\end{array}$ & 305 \\
\hline 24 & $\begin{array}{l}\text { Extraindo as raízes dos graus, minutos e ordens } \\
\text { inferiores }\end{array}$ & 307 \\
\hline 26 & $\begin{array}{l}\text { Sobre como resolver tudo o que é necessário da } \\
\text { aritmética do hindi sem as nove letras }\end{array}$ & 310 \\
\hline 27 & $\begin{array}{l}\text { Por Hindi: Escritos, Nomes, Cálculos - Tudo que } \\
\text { Queremos }\end{array}$ & 312 \\
\hline 28 & $\begin{array}{l}\text { Cálculo (ferramenta) para cegos e deficientes visuais } \\
\text { Extração do lado do cubo }\end{array}$ & 313 \\
\hline 30 & $\begin{array}{l}\text { Sobre o conhecimento de frações que são cúbicas e a } \\
\text { extração de seus lados, simples ou combinados em } \\
\text { unidades }\end{array}$ & 328 \\
\hline 31 & Extraindo os lados de números que não têm raízes & 332 \\
\hline 32 & Em Dobrar Um, Sessenta e Quatro Vezes & 337 \\
\hline
\end{tabular}

Fonte: Adaptado de Al-Uqlidisi (1978)

A organização da obra em cada um dos livros propostos foi pensada de forma lógica de maneira que, além de disseminar os conhecimentos já existentes, estes pudessem ser adaptados para a utilização de ferramentas que trouxessem rapidez e praticidade. De fato, propor novos procedimentos utilizando os algarismos indianos requeria conhecer bem esse sistema e os demais que estavam em uso no período.

Dessa maneira, no primeiro livro o autor aborda, segundo seus escritos, o necessário para a compreensão da aritmética que adotava os símbolos indianos. AlUqlidisi(1978) informa, ao introduzir este livro, que poderia substituir todas as demais obras desse conteúdo já existentes até então (século X). As explicações do autor islâmico têm início com a apresentação dos nove símbolos indianos a as características do sistema de numeração para a realização dos cálculos em um ábaco de areia.

O Instrumento mencionado no texto histórico demandava o uso de uma espécie de vareta para desenhar os números e as mãos eram utilizadas para apagar os registros parciais dos cálculos, sendo que os números ocupavam os lugares das unidades, dezenas, centenas e assim respectivamente. Após essa preliminar são explicadas as técnicas para a 
duplicação e redução pela metade nos números, multiplicação, divisão, multiplicações diversas com frações além da extração de raízes, operações com graus e minutos.

Após a base fornecida, o segundo livro é descrito pelo autor como fonte de ideias mais avançadas e curiosidades sobre o trabalho com os números grandes (com muitas ordens), difíceis de trabalhar no Rumi. De acordo com Saidan (1978) esse sistema corresponde ao cálculo mental utilizando o movimento dos dedos das mãos, praticado pelos árabes bizantinos, de origem derivada do império romano.

As informações expostas indicam que Al-Uqlidisi estava adaptando métodos de outras culturas para a aplicação dos símbolos indianos. Ressalta-se que os conteúdos são semelhantes aos do primeiro livro, mas são atribuídos procedimentos diferenciados. Encerrando assim a exposição dos conhecimentos aritméticos antigos.

A partir disso, no terceiro livro, o Euclidiano tece um jogo de perguntas e respostas gerado por questionamentos direcionados que pairavam sobre os calculistas, inclusive explica porque traz primeiro um procedimento e depois outro. Simplesmente, pela preocupação do autor estar em o leitor compreender melhor o procedimento. A título de exemplo tem o caso que Al-Uqlidisi (1978) apresenta o motivo de a duplicação (dobrar) vir antes da multiplicação por ser um caminho mais fácil para aprender a multiplicar.

Esclarecidas as dúvidas na adoção do sistema da aritmética indiano, no quarto livro o autor medieval faz a transferência do método de cálculo com as técnicas indianas no ábaco de areia para o uso desses conhecimentos escritos no papel. Al-Uqlidisi aponta o desconforto na utilização do instrumento indiano, tendo em vista que ele não guardava os resultados, no caso da ação do vento, por exemplo. O calculista teria que refazer as contas, além da necessidade do apagamento para a reutilização do artefato. No papel, o não apagamento das contas tornou os cálculos mais ágeis além de evitas sujar as mãos com a areia.

Esse capítulo marca de forma significativa a contribuição islâmica a partir da aritmética indiana. Além de trazer os conteúdos da duplicação, redução, multiplicação, divisão com frações, extração de raízes e demais que aparecem nos outros capítulos nessa nova abordagem, Al-Uqlidisi mostra ainda como trabalhar sem a utilização dos símbolos indianos em um capítulo e mostra um artefato construído em madeira ou marfim para que as pessoas cegas possam se beneficiar dos conhecimentos apresentados, cabendo aprofundamentos futuros. 


\section{Algumas Considerações}

Al-Uqlidisi, ao publicar o Kitab al-Fusul fi al-Hisab al-Hindi proporcionou a divulgação dos conhecimentos aritméticos indianosque foram incorporados na cultura islâmica e as trasformações ocorridas neles que redimensionaram as práricas de cálculo com os benefícios da escrita.

Entretanto, no texto não foram explicitados que ofícios na sociedade de Damasco da época em estudo recorriam a tais conhecimentos matemáticos, cabendo assim um aprofundamento da análise contextual histórica mais ampla de forma que se estabeleça conexões entre tais concepções e a sociedade em que elas foram difundidas.

Esta primeira etapa da pesquisa poderá subsidiar estudos futuros no sentido de compreender a lógica interna do documento explorado, fornecendo elementos com potencial para ressignificar o entendimento dos procedimentos modernos de cálculos artiméticos, com vistas a elaboração de propostas para incorporação de elementos da história no ensino.

\section{Referências}

ABATTOUY, M. Ahmad Salim Sa'idan: A Palestinian Historian of Arabic Mathematics. Disponível em: https://muslimheritage.com/ahmad-salim-saidanpalestinian-historian-arabic-mathematics/. Acesso em 27 de fev. de 2021 às 10h 20min.

AL-UQLISISI. Kitab AL-fusul fi AL-hisab AL-hindi. In: SAIDAN, A. S. The arithmetic of Al-Uqlidisi: the story of hindu-arabicaritmétic as told in Kitab al-fusul fi al-hisab al- híndi. 1 ed. Boston: Reidel Publishing Company, 1978.

AL-UQLISISI. Kitab AL-fusul fi AL-hisab AL-hindi. Istambul: Biblioteca Suleymaniye, manuscrito n. 802, 1186. Disponível em: < https://ima.diamondils.org/work/view/4001>. Acesso em 15 de fev. de 2020 às $20 \mathrm{~h}$.

ABEL. R. À propos de l'originegrecquedeschiffres de Fès et de noschiffresarabes. RevuedesÉtudesGrecques, n. 48, 1935, pp. 525-539. Disponível em: <https://www.persee.fr/doc/reg_0035-2039_1935_num_48_228_4962>. Acessoem 01 de fev. de 2021 às $08 \mathrm{~h} 30 \mathrm{~min}$.

SAIDAN, A. S. The arithmetic of Al-Uqlidisi: the story of hindu-arabicaritmétic as told in Kitab al-fusul fi al-hisab al- híndi. 1 ed. Boston: Reidel Publishing Company, 1978.

SAIDAN, A. S. The Earliest Extant Arabic Arithmetic: Kitab al-Fusul fi al Hisab alHindi of Abu al-Hasan,Ahmad ibn Ibrahim al-Uqlidisi. Isis, vol. 57, n. 4, pp. 475-490, 
1966. Disponível em: <http://www.jstor.org/stable/228518>. Acesso m 20 de nov. de 2020 às $10 \mathrm{~h} 30 \mathrm{~min}$.

SAITO, Fumikazu. História da matemática e suas (re)construções contextuais. São Paulo: Livraria da Física, 2015.

TUNER, H. R. Science in medieval Islã: an illustrated introduction. Austin: University of Texas Press, 2006.

Recebido em: 27 / 04 / 2021

Aprovado em: 15 / 04 / 2021 